\title{
Hertz Per Second
}

National Cancer Institute

\section{Source}

National Cancer Institute. Hertz Per Second. NCI Thesaurus. Code C154854.

A unit of frequency rate change defined as the number of Hertz per unit of time equal to one second. 